\title{
Construção de um jogo educativo em uma plataforma de desenvolvimento de jogos e aplicativos de baixo grau de complexidade: o caso do Quizmica - Radioatividade
}

\author{
Cicero Ernandes de Melo Santos, Bruno Silva Leite \\ Laboratório para Elaboração e Utilização das Tecnologias no Ensino de Química \\ Universidade Federal Rural de Pernambuco \\ cicero.ernandes@ufrpe.br, leitebrunosilva@gmail.com
}

Resumo: Observa-se atualmente uma forte tendência de uso das tecnologias digitais como subsídios na construção do conhecimento. A utilização de jogos digitais, na educação, tem contribuído para o processo de ensino e aprendizagem. Dentre os diversos tipos, o Quiz é um gênero de jogo bem difundido entre os usuários da web. Nesse sentido, o objetivo deste trabalho é apresentar o projeto e o desenvolvimento de um jogo digital do tipo Quiz que cadencia a aprendizagem sobre Radioatividade e entretenimento. Para a elaboração do Quiz optou-se pelo MIT App Inventor por ser uma plataforma gratuita de desenvolvimento de jogos e aplicativos de baixo grau de complexidade. Além disso, esta pesquisa mostra os aspectos técnicos e demais propriedades do jogo Quizmica Radioatividade que tem como intuito contribuir para a compreensão dos conteúdos de radioatividade, proporcionando uma aprendizagem mais ativa sobre o assunto proposto, possibilitando que os estudantes façam uso do aplicativo de modo ubíquo.

Palavras-chave: Jogo Digital, Ensino de Química, Radioatividade, Quiz.

\section{Construction of an educational game in a platform for developing games and applications of low degree of complexity: the case of Quizmica - Radioatividade}

\begin{abstract}
Today there is a strong tendency to use digital technologies as subsidies in the construction of knowledge. The use of digital games in education has contributed to the teaching and learning process. Regarding game types, the Quiz is a widespread game gender among web users. In this sense, this paper aims at presenting the design and the development of a digital game, a kind of Quiz, that harmonize both learning of Radioactivity and entertainment. For the preparation of the Quiz, we chose MIT App Inventor as a free platform for developing games and applications of the low degree of complexity. In addition, this research shows the technical aspects and other properties of the Quizmica - Radioatividade game that aims to contribute to the understanding of radioactivity contents, providing more active learning on the proposed subject, allowing students to use the application in a ubiquitous way.
\end{abstract}

Keywords: Digital Game, Chemistry Teaching, Radioactivity, Quiz.

\section{Introdução}

O rápido crescimento da tecnologia digital nas últimas décadas mudou a forma com que os estudantes veem o ambiente educacional no qual estão inseridos. Observa-se nas escolas uma nova geração dinâmica e inovadora, chamada de geração Z (zapear/zap) (Marques; Marques, 2016). Nesta geração os estudantes estão sempre conectados à internet e fazem tudo de maneira rápida, além de conviverem com a tecnologia diariamente realizando diversas tarefas ao mesmo tempo. Nesse contexto, considera-se adequada a busca por metodologias alternativas de caráter inovador que os auxiliem no processo de ensino e aprendizagem. Para esta geração o uso de métodos de ensino atualizados e voltados aos seus interesses são importantes, pois não há como seguir as 
mesmas metodologias, a necessidade de inovação se faz necessária (Marques; Marques, 2016).

Os Recursos Didáticos Digitais (RDD) com potencial para serem utilizados na educação surgem cada vez mais associados aos dispositivos móveis (DM), em sua grande maioria são apresentados na forma de aplicativos (Apps) para tablets e smartphones. Os RDD na educação contribuem para o uso de diferentes estratégias no processo de ensino e aprendizagem e podem ajudar professores e estudantes a encontrarem formas distintas de aprenderem um determinado conteúdo (Leite, 2015). O potencial dos dispositivos móveis deve-se, em grande parte, a imensa variedade de funções próprias destes aparelhos, bem como das possibilidades decorrentes da instalação de novos aplicativos.

O desenvolvimento de Apps tem crescido nos últimos anos e em amplo destaque temos a produção de diversos tipos de games. Segundo Prensky (2003) os games contribuem para a criação de um amplo ficcional, que oferece novas formas de entender e agir sobre o real. Eles podem ser reconhecidos como facilitadores da aprendizagem de assuntos complexos e por desenvolver importantes habilidades cognitivas, como a resolução de problemas, a percepção, a criatividade e o raciocínio rápido.

Com o surgimento de plataformas para o desenvolvimento de games de fácil utilização tem-se ampliado as possibilidades para que professores, com pouco conhecimento de programação, se interessem pelo desenvolvimento de jogos e aplicativos educacionais, tirando esse encargo das mãos (antes exclusivamente) dos programadores, como vinha ocorrendo até então (Tarouco et al., 2004). Neste sentido, o presente trabalho tem como objetivo descrever o processo de desenvolvimento de um protótipo de jogo educativo denominado de Quizmica - Radioatividade, a partir do uso de uma plataforma para elaboração de aplicativos com baixo grau de complexidade.

\section{O conteúdo de Radioatividade no Ensino de Química}

A Química e a Física Nuclear podem ser entendidas como uma ciência Nuclear, a junção das pesquisas advindas dessa ciência tornou possível o conhecimento da natureza da matéria, tendo por consequência acarretado em benefícios para diversas áreas, tais como a medicina, eletrônica, geologia e as indústrias (Tekin; Nakiboglu, 2006). No entanto, poucos professores de química do ensino médio abordam a química nuclear e em especial a radioatividade em suas aulas, seja por limitação em sua formação inicial ou pelo fato de que autores de livros e editoras não dão o mesmo destaque que é dado para outros assuntos (Tekin; Nakiboglu, 2006).

Sendo um fenômeno natural a radioatividade consiste na emissão espontânea de partículas e/ou radiação por átomos que apresentam núcleos instáveis. Por meio de diversos estudos ao longo dos anos, os fenômenos radioativos puderam ser controlados e produzidos artificialmente e utilizados em diferentes finalidades, tais como diagnósticos por imagem, esterilização de materiais cirúrgicos, radioterapia, entre outros (Fernandes; Campos, 2016). Constantemente, assuntos recentes são associados a radioatividade como os casos de energia, meio ambiente, acidentes nucleares e lixo radioativo, além disso este campo de conhecimento é de suma importância para compreensão da estrutura do átomo, abrangendo diversas áreas no ensino de Ciências, tais como a Física e Química (Silva et al., 2013).

O conteúdo sobre radioatividade é parte integrante dos currículos do ensino médio e superior de Química no Brasil, é um assunto contemporâneo sendo recomendado nas orientações curriculares oficiais (Brasil, 2006). Todavia, percebe-se que este conteúdo ainda é tímido nas pesquisas envolvendo o ensino de Química, apresentando escassos trabalhos e propostas metodológicas para o processo de ensino e aprendizagem (Silva et al., 2013), principalmente no que se refere ao uso de jogos digitais. Diante deste quadro, 
entende-se a importância do uso de jogos digitais no ensino de Química e das possibilidades de aplicações destes no processo da construção do conhecimento químico que contemplassem o conteúdo de radioatividade.

\section{Potencialidades dos jogos e aplicativos para o Ensino de Química}

Os jogos estão intimamente ligados como parte da nossa vida desde os tempos mais remotos, estando presentes não só na infância, mas em diversos momentos. Estes podem ser recursos de aprendizagem eficientes, pois possuem a capacidade de divertir, enquanto motiva, de facilitar o aprendizado e aumentar a capacidade de retenção do que foi ensinado exercitando as funções mentais e intelectuais de quem joga (Tarouco et al., 2004). Fazer o uso destas ferramentas em um contexto educacional pode tornar-se uma abordagem eficiente e atrativa no processo de ensino e aprendizagem.

Diante da evolução dos recursos tecnológicos e eletrônicos, temos uma nova categoria de jogos com aspectos lúdicos, ficcionais e imersivos que são os jogos digitais ou games (Ghensev, 2010). Compreendendo que game e jogo podem ter o mesmo significado (Leite, 2017), conjectura-se que os games se encontram dentro de uma categoria maior que são os jogos, assim muitas características encontradas nos jogos "tradicionais" estão presentes nos games. Contudo, há outras características relevantes que os games propiciam ao jogador, como o ambiente controlado, o dinamismo, a forma como as regras são apresentadas e impostas, junção de som, imagem e texto, criando uma imersão e realidade atingidas somente pelo auxílio da tecnologia digital (Kruger; Cruz, 2001).

Considerando que os jogos digitais podem ser utilizados diretamente no contexto educacional, dentro de uma estratégia que favoreça o processo de ensino e aprendizagem, sua utilização pode promover importantes contribuições. Ao interagir com os jogos, as funções cognitivas são intensificadas, proporcionando ao jogador diferentes formas de construir seu conhecimento (Santos; Alves, 2016).

Com a ascensão exponencial dos dispositivos móveis com acesso à internet (Ex.: smartphones e tablets), estes tornam-se cada vez mais popularizados e difundidos. É bastante comum que estudantes utilizem os DM cotidianamente para acessar as redes sociais, fazerem buscas na internet e também para jogarem jogos digitais. Observa-se que os DM podem proporcionar aos professores e estudantes mobilidade e interface fácil de usar, podendo assim, contribuir para implementar diferentes estratégias de ensino e de aprendizagem, ampliando as possibilidades de ação e interação entre sujeitos, sujeitos e meio. Ademais, a popularização das tecnologias móveis tem impulsionado o desenvolvimento de novos aplicativos com potencial para incrementar o aprendizado dentro e fora da sala de aula (Nichele; Schlemmer, 2014).

Considerando a importância da inovação em práticas pedagógicas e que as tecnologias digitais estão intimamente ligadas ao cotidiano dos estudantes, é pujante fazer uso dos apps no processo de ensino e aprendizagem. Para o ensino de Química observa-se diversos tipos de apps disponíveis nas plataformas digitais (ex: Google Play e App Store). A utilização de aplicativos em um contexto educacional permite novas possibilidades, de modo que a aprendizagem auxiliada por estes recursos digitais propicia uma interação a mais, podendo incrementar o ensino dentro e fora da sala de aula (Marques; Marques, 2016).

Em relação as potencialidades oferecidas pelos jogos digitais e aplicativos, Nichele e Schlemmer (2014) elencaram alguns apps com potencial para serem utilizados no processo de ensino e aprendizagem de Química (Tabela 01). 
Tabela 1 - Aplicativos que podem ser utilizados no ensino de Química.

\begin{tabular}{|c|c|}
\hline Aplicativos & Sobre \\
\hline Merck PTE HD & $\begin{array}{r}\text { Tratam-se de Apps que essencialmente fornecem uma tabela } \\
\text { periódica para consulta interativa de dados. }\end{array}$ \\
\hline Elements - Periodic Table & $\begin{array}{c}\text { São exercícios apresentados na forma de jogos. Neste game o } \\
\text { usuário é desafiado a relacionar e comparar a posição de } \\
\text { elementos com suas propriedades periódicas. }\end{array}$ \\
\hline Tabela Periódica Quiz & $\begin{array}{c}\text { Game no qual o usuário e desafiado a relacionar nomes de } \\
\text { elementos a seus respectivos símbolos. }\end{array}$ \\
\hline Moléculas & $\begin{array}{c}\text { Permite rotacionar, mover e dar zoom para várias moléculas } \\
\text { (cerca de 270) que estão disponíveis na biblioteca do aplicativo. } \\
\text { A busca por essas moléculas pode ser realizada pelo nome ou pela } \\
\text { fórmula molecular. }\end{array}$ \\
\hline ChemSpider Mobile & $\begin{array}{c}\text { Permite visualizar a representação de moléculas e suas estruturas } \\
\text { bidimensionais de sua biblioteca e outros dados. }\end{array}$ \\
iMolview Lite & $\begin{array}{c}\text { Permite visualizar diferentes estruturas e "manipulada" } \\
\text { (rotacionada, ter seu tamanho aumentado ou diminuído). }\end{array}$ \\
\hline
\end{tabular}

Fonte: adaptado de Nichele e Schlemmer (2014)

A partir deste levantamento, Nichele e Schlemmer (2014) alertam que a utilização de softwares e dispositivos móveis para fins de ensino precisa ser planejada de modo que as atividades desenvolvidas possam ir além de uma mera transposição de conteúdo do meio analógico para o digital. Neste contexto, na escolha de um aplicativo é importante averiguar o quanto ele atende às necessidades do professor e dos estudantes, por exemplo: ler a descrição dos aplicativos e os comentários de outros usuários; conferir o site do aplicativo e verificar se os desenvolvedores atuam em uma área específica; experimentar o aplicativo antes de utilizá-lo em sala de aula.

Destaca-se que na pesquisa de Nichele e Schlemmer (2014) e em nosso levantamento nas plataformas digitais, não foram encontrados nenhum jogo/aplicativo que envolvesse os conteúdos de radioatividade com objetivos pedagógicos.

\section{O jogo Quizmica - Radioatividade}

$\mathrm{Na}$ tentativa de construir um jogo digital que apresentasse um ponto de equilíbrio entre entretenimento e aprendizagem de conceitos químicos, aceitamos o desafio de desenvolver o jogo Quizmica - Radioatividade de forma que este jogo constituísse em um espaço para a aprendizagem da Radioatividade. Na presente seção, apresentamos os resultados alcançados durante o processo de elaboração do Quizmica - Radioatividade. Primeiramente destacamos o projeto do jogo, em seguida relatamos o processo de desenvolvimento do App e por fim o produto final.

\subsection{O projeto}

O jogo digital Quizmica - Radioatividade é parte de um projeto de pesquisa desenvolvido em uma universidade federal pública que elabora e valida o uso de recursos didáticos digitais no ensino de Química. Para o desenvolvimento do protótipo, inicialmente foi realizado um levantamento de jogos digitais disponíveis na loja virtual Google Play, na qual constatou-se que o conteúdo sobre radioatividade até o momento desta pesquisa não havia sido explorado para fins pedagógicos, disponibilizando apenas softwares com enfoques em histórias de acidentes nucleares, música, comédia, wallpaper e simuladores de detecção de radiação. Destarte, sucedeu de escolhermos este assunto para a elaboração do nosso jogo, que é baseado na clássica mecânica de perguntas e respostas conhecido como "Quiz", contendo pontuações ao longo do jogo. 
A modalidade do jogo digital Quiz basicamente consiste em perguntas e respostas que visam estimular o aprendizado por meio de recursos presentes nos softwares como desafios e progressão, possibilitando a imersão e interação entre os participantes, considerando-se ainda a função lúdica que está presente nessa modalidade. Esse tipo de jogo é observado nas mais diversas formas de entretenimento, tais como no rádio, em programas de TV, nos softwares etc. Segundo Germano et al. (2011) as vantagens de utilizar os Quizzes é que eles possibilitam um feedback imediato na revisão de conceitos abordados nas perguntas relacionadas ao conteúdo discutido e podem ser um elemento motivador quando utilizados juntamente com aulas expositivas.

Compreendendo-se o potencial desta modalidade de jogo no ensino e considerando que a Química, para a maioria dos discentes, é uma disciplina vista como de difícil entendimento (Souza et al., 2015), em alguns casos com baixo rendimento na aprendizagem, o projeto "Quizmica" pretende contribuir para a melhoria no processo de construção do conhecimento químico dos estudantes.

\subsection{O desenvolvimento}

Cientes desde o início do projeto quanto à necessidade de trabalhar o conteúdo sobre Radioatividade, resolvemos percorrer um caminho objetivo durante sua produção. A prioridade seria elaborar um jogo dinâmico, atrativo, com feedback em tempo real, de modo que em sua execução pudesse conquistar o jogador e dentro deste contexto, evidenciar o conteúdo Químico, sem que se perdesse a interação e o desejo de jogar. Nessa perspectiva, Mattar (2010) afirma que uma das grandes dificuldades no processo de aprendizagem é que a escola separa aprendizagem de prazer, por isso buscamos no desenvolvimento do App propiciar a interação com um jogo divertido e através dele, mediar a aprendizagem. Tais definições foram subsidiadas pela análise dos jogos indicados na seção 3 e pela nossa imersão no universo dos games.

Segundo Tarouco et al. (2004) para o desenvolvimento de jogos educacionais primeiramente deve-se definir alguns elementos essenciais, tais como: o tema a ser abordado; quais os objetivos a serem alcançados com o software; escolha e produção das mídias que serão parte do jogo (como imagens, áudios e textos); a forma que será feita a organização; etc. Após estas definições, o jogo é desenvolvido através de um linguagem de programação.

De modo geral, diversos tipos de games são desenvolvidos diariamente para algum tipo de finalidade. A criação destes, na grande maioria das vezes, é essencialmente feita por programadores com vasto conhecimento na área. Para estes é imprescindível o conhecimento de conceitos relacionados à linguagem de programação, algoritmo, edição de imagens digitais e plataforma de programação de games, entre outros.

Com o surgimento de ambientes de desenvolvimento de softwares cada vez mais intuitivos e de baixo grau de complexidade, tem-se facilitado o acesso para que professores e pedagogos com pouca experiência na área de programação de games criem seus próprios projetos. Nesse contexto, entende-se como plataformas de baixo grau de complexidade de desenvolvimento de softwares ambientes no qual o usuário não necessita necessariamente saber programar, nem saber uma linguagem de programação para poder desenvolver seus próprios games, pois estas plataformas dispensam a digitação manual de scripts. Estas plataformas, em alguns casos, são acompanhadas com comandos previamente prontos necessitando apenas que o usuário utilize-as de acordo com a demanda de seu projeto ou de forma alternativa a tradicional (digitação manual) a partir do estilo de programação drag-and-drop (arrastar-soltar).

A diversidade de ambientes de desenvolvimento de softwares cada vez mais simples e intuitivos tem facilitado o processo de desenvolvimento de jogos educacionais, 
propiciando que professores deixem o estado de meros expectadores e avaliadores para desenvolvedores de games e aplicativos educacionais, podendo desenvolver jogos digitais eficientes e contextualizados com a sua realidade (Tarouco et al., 2004). Neste sentido, buscou-se desenvolver um protótipo de jogo digital de perguntas e respostas utilizando uma plataforma de baixo grau de complexidade.

A plataforma de desenvolvimento escolhida para a elaboração do jogo foi o MIT App Inventor, devido a seus recursos e sua interface simples e intuitiva, permitindo a criação de aplicativos para smartphones e tablets que fazem uso do sistema operacional Android. A escolha deste sistema operacional para a primeira versão do aplicativo se justifica pelo fato de ser um dos mais utilizados no mercado de produção de Apps para dispositivos móveis (Businesswire, 2017), por suas diversas funções e utilidades, sendo considerado o mais popular até o pressente momento.

Contendo diversos recursos, o MIT App Inventor é uma plataforma de código aberto que foi desenvolvida conjuntamente pela Google e pelo Massachusetts Institute of Technology (MIT). Seu funcionamento ocorre por meio da nuvem, sendo possível acessar por meio de qualquer tipo de navegador (Google Chrome, Firefox, Baidu Browser etc.). De característica simples e intuitiva, a programação nesta plataforma é baseada de maneira análoga a um quebra-cabeça juntando peça por peça, em que é feita a adição de elementos (tais como botões, animações e caixas de texto) por meio de encaixe em "blocos". Cada ação realizada especifica como os componentes devem se comportar e ao final de cada projeto é possível fazer o download do App criado e instalar nos DM que fazem uso do sistema operacional Android. Além disso, é possível disponibilizar o App na loja virtual da Google Play. No site do MIT App Inventor são disponibilizados diversos estudos que abordam desde a instalação até a criação do primeiro aplicativo. Além disso, são disponibilizados relatos de experiências de professores e estudantes no uso desta plataforma (Gomes; Melo, 2013).

Para a elaboração do jogo no MIT App Inventor é preciso que o usuário acesse a plataforma online e vincule uma conta do Gmail para ter acesso à interface de criação de projetos. Um dos primeiros passos para a elaboração do Quizmica - Radioatividade foi a estruturação das telas do jogo (12 telas ao todo) seguido da inserção dos botões e caixas de texto (Figura 1A). Na parte da programação, um dos principais itens que um Quiz deve ter é o banco de dados para armazenar as perguntas e respostas, assim foi utilizada a função TyniDB seguida de arranjos em tabelas (Figura 1B) para que as perguntas e pontuações permanecessem armazenadas de maneira offline (sem a necessidade de internet no DM do usuário).

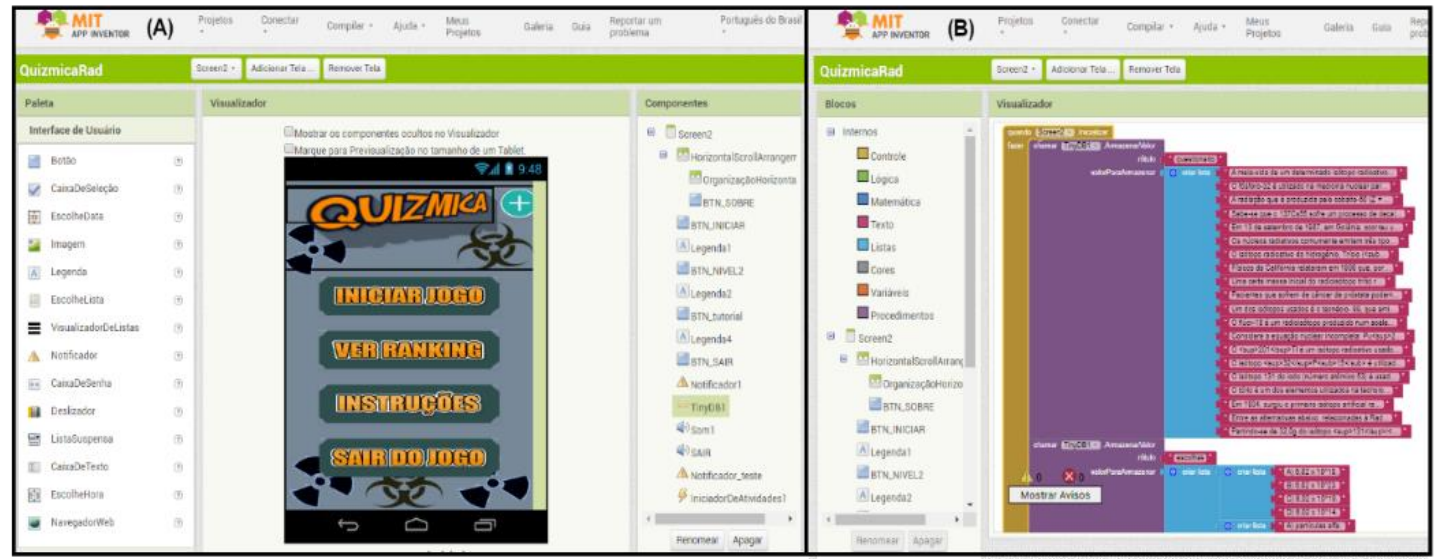

Figura 1 - (A) Tela de desenvolvimento do App. (B) Tela de programação do App. 
Outros algoritmos foram utilizados para o funcionamento do jogo, no entanto por limitações de espaço optamos por mostrar as principais interfaces no desenvolvimento do App nas figuras $1 \mathrm{~A}$ e $1 \mathrm{~B}$.

A partir da escolha do MIT App Inventor como plataforma de desenvolvimento do jogo, utilizamos para a criação e edição dos layouts contidos no aplicativo o software Gimp (versão 2.8). Esse software dispõe de diversos recursos para edição e manipulação de imagens digitais, bem como o fato de ser um software gratuito, atendendo as necessidades do projeto.

\subsection{O produto final}

O jogo Quizmica - Radioatividade conta com uma interface extremamente intuitiva e de fácil utilização. Seu nome vem do acrônimo das palavras Quiz e Química. A ideia de criar um Quiz digital surgiu da necessidade de ter um recurso simples e intuitivo para auxiliar no estudo da radioatividade e que apresentasse caráter desafiador, prático e motivador, pressupostos da gamificação (Leite, 2017), possibilitando que aprendizagem ocorresse a qualquer momento e em qualquer lugar, aproveitando-se das vantagens dos DM no tocante a mobilidade espacial e temporal. O jogo digital aborda os conteúdos envolvendo radioatividade, ao ser iniciado o mesmo exibe uma tela de apresentação que mostra algumas informações acerca de seu desenvolvimento (Figura 2A), posteriormente em sua tela principal apresenta cinco botões: "Iniciar jogo", "Ver ranking", "Sobre" (representada pela seta +), "Instruções" e "Sair do jogo" (Figura 2B). Ao selecionar "Iniciar jogo" uma nova tela apresenta três modos de jogo representados pelos cientistas Antoine Henri Becquerel, Ernest Rutherford e Marie Curie. A escolha destes cientistas se deve as suas contribuições na elucidação da Radioatividade. Cada cientista representa um nível distinto de dificuldade, sendo Becquerel o nível mais fácil e Curie o nível mais complexo (Figura 2C).
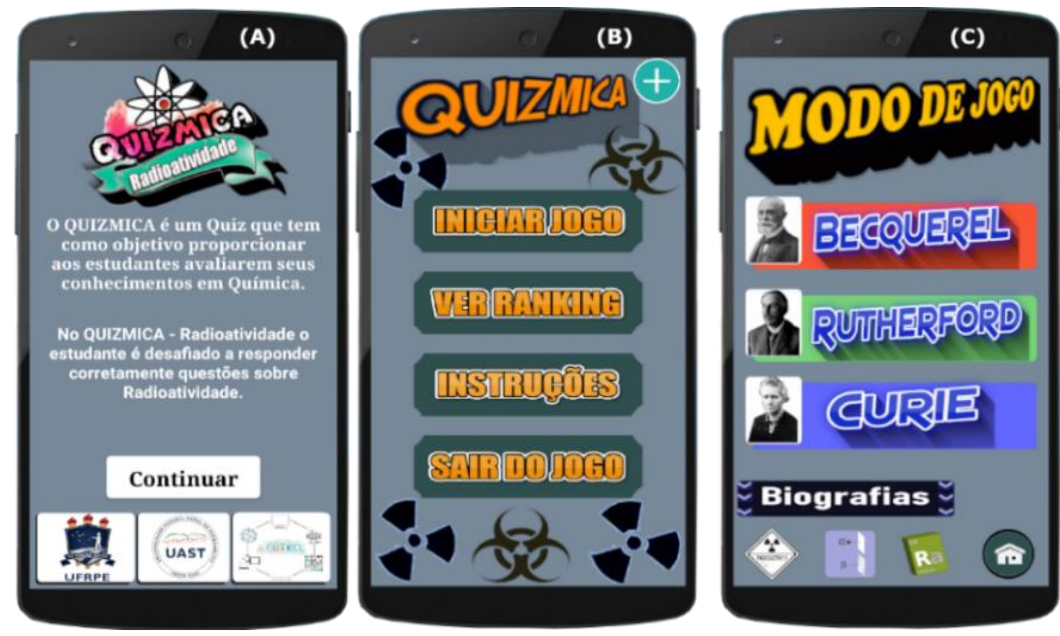

Figura 2 - (A) Tela de apresentação. (B) Menu inicial. (C) Tela de níveis.

Ainda na tela "modo de jogo" (Figura 2C) é possível encontrar três botões com imagens que fazem referência a cada cientista (logo abaixo da palavra "Biografias"), na qual ao serem clicados fazem surgir uma tela contendo informações a respeito da vida do cientista selecionado (Figura 3). A primeira imagem remete à ideia da radioatividade (Becquerel), a segunda as partículas alfa e beta (Rutherford) e a terceira ao símbolo do elemento químico Rádio (Curie). Estas imagens corroboram para que seja observado como cada cientista contribuiu para os conhecimentos que serão discutidos no Quiz, sendo uma forma de estimular a curiosidade de cada jogador. 


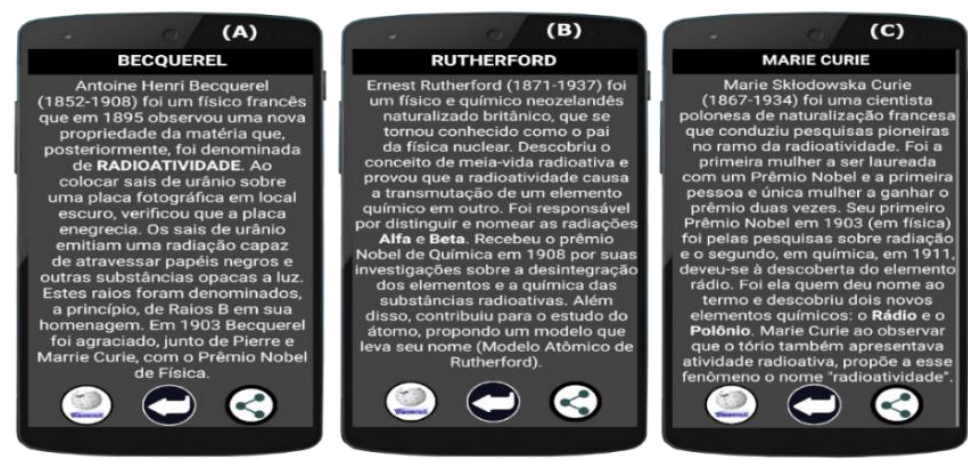

Figura 3 - Biografia de Becquerel (A), Rutherford (B) e Marie Curie (C).

Cada modo de jogo (Figura 2C) possui um número de perguntas com quatro alternativas e apenas uma resposta correta. Destarte, o primeiro modo (Becquerel) contém 10 perguntas, o segundo (Rutherford) 15 perguntas e o terceiro (Curie) 20 perguntas. Cabe destacar, que as perguntas dos modos não são idênticas, totalizando 45 perguntas distintas do Quiz. A cada resposta correta o jogador recebe dez pontos (Figura 4A), para as incorretas não há pontuação. Ao final de cada modo, o jogador poderá ver sua pontuação e sua colocação (Figura 4B) clicando no botão "Ver Ranking" (Figura 2B).

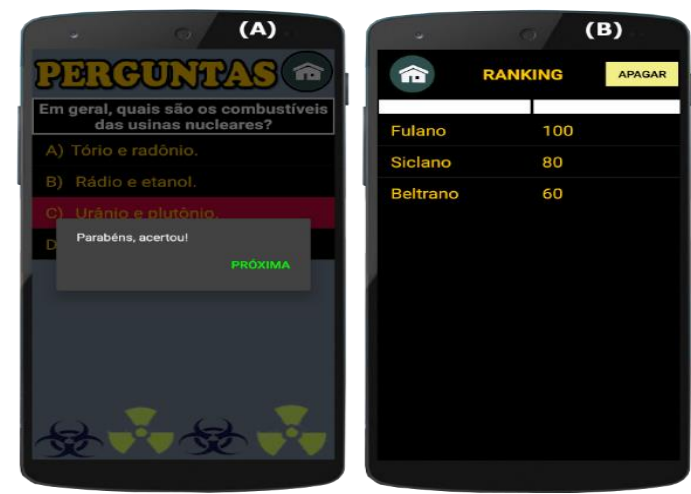

Figura 4 - (A) Tela de perguntas e respostas. (B) Tela de Ranking dos jogadores.

Após a primeira etapa de nossa pesquisa (elaboração do jogo), pretendemos propor estratégias didáticas para a utilização e validação deste RDD nos ambientes educacionais. Almejamos, por exemplo, implementar o jogo como uma atividade extra sala com desafios para o engajamento dos estudantes, incentivar seu uso em atividades para revisar os conceitos de radioatividade, promover campeonatos entre grupos de estudantes etc.

De modo a avaliar a eficácia do game e suas atribuições (design, modo de jogo, dificuldade, ludicidade etc.), bem como sua usabilidade e identificar possíveis erros conceituais e técnicos, o Quizmica - Radioatividade foi utilizado por seis (6) professores de Química de três universidades federais do Brasil e nove (9) licenciandos em Química (do grupo de pesquisa que o projeto está sendo desenvolvido). O feedback dos professores e estudantes indicam prospectivamente que o jogo Quizmica - Radioatividade apresenta uma boa qualidade para a finalidade educativa. Logo, a avaliação por tais participantes se torna um elemento chave para darmos seguimento a próxima etapa da pesquisa (aplicação e validação com alunos do ensino médio). Nesse sentido, o app será apropriado por professores de Química (do ensino básico e superior), professores em formação (licenciandos em Química) e por estudantes do ensino básico, através do método GameFlow (Jerges, 2007).

Considerando que os estudantes geralmente estão conectados à internet e convivem com a tecnologia diariamente (Marques; Marques, 2016), além das possibilidades que as 
tecnologias digitais apresentam no cenário educacional e social, práticas de ensino aliadas ao uso de games e aplicativos se configuram como uma estratégia relevante ao cotidiano da chamada geração $\mathrm{Z}$, de modo que o uso destes recursos possibilitam um rompimento com o paradigma tradicional de ensino (Tarouco et al., 2004). Nesse contexto, acreditamos que é importante discutir o quanto um aplicativo voltado para o ensino de Química pode contribuir em relação ao conhecimento científico na formação do estudante e como o conhecimento adquirido durante o Quizmica - Radioatividade contribui para romper com as práticas tradicionais, por muitas vezes enraizadas nas aulas de química.

\section{Considerações finais}

A utilização de Recursos Didáticos Digitais, tais como smartphones e tablets, associados aos games e aplicativos podem propiciar experiências positivas no processo de ensino e aprendizagem, tendo em vista que quando bem aproveitadas pode ocasionar em maior engajamento, motivação e consequentemente melhores resultados escolares. Dessa forma, acreditamos que com o surgimento de plataformas de baixo grau de complexidade de desenvolvimento de games, os aplicativos educativos desenvolvidos por professores podem ser mais intuitivos, eficientes e ótimas ferramentas auxiliadoras no processo de ensino e aprendizagem, tendo visual e recursos contextualizados e propícios para que ocorra uma aprendizagem significativa.

Constituído de diversos recursos como ranking, níveis e pontuação, a elaboração do jogo educativo Quizmica - Radioatividade (de temática ainda não explorada até o pressente momento) objetiva contribuir para a compreensão dos conteúdos de radioatividade em que os estudantes possam fazer uso do aplicativo de modo ubíquo (uma das características presentes na aprendizagem móvel), demonstrando sua potencialidade como um RDD. Além disso, de maneira propedêutica apresenta o quão acessível pode ser o desenvolvimento de games educativos por professores e estudantes sem a necessidade de um domínio específico em linguagem de programação.

Por fim, as contribuições que os jogos digitais podem proporcionar para o processo de ensino aprendizagem da Química são inúmeras, eles fazem parte do cotidiano de nossos alunos e como educadores precisamos aprender a lidar com eles. Ademais, é preciso utilizarmos as potencialidades dos jogos digitais, aproximando a escola do universo dos alunos. Para tanto, o jogo Quizmica - Radioatividade foi desenvolvido com o intuito de contribuir nesta área de pesquisa, sendo um ambiente lúdico e divertido, onde é possível abordar um conteúdo de Radioatividade sem ser maçante e desmotivante.

Atualmente o jogo está disponível na Google Play para download gratuito (http://bit.ly/2GW9wSF) e está em desenvolvimento um material suplementar com o intuito de dar suporte ao professor na utilização do jogo (com sugestões de estratégias e sequências didáticas para seu uso em sala de aula). Nossa perspectiva é contribuir para esta área de pesquisa apresentando, em futuras publicações, dados que demonstrem tais contribuições dos jogo digitais no contexto educacional da Química.

\section{Referências}

BRASIL. Secretaria de Educação Básica. Orientações Curriculares Nacionais. Brasília: Ministério da Educação, 2006.

BUSINESSWIRE. Android Overtakes Windows for First Time - StatCounter. 2017. Disponível em: <https://tinyurl.com/y5fl2fn8>. Acesso em: 18 de abr. 2019. 
FERNANDES, L. S; CAMPOS, A. F. Análise das questões sobre radioatividade no Exame Nacional do Ensino Médio-ENEM. Amazônia: Revista de Educação em Ciências e Matemáticas, v. 13, n. 25, p. 62-74, 2016.

GERMANO, A. S. M; AROCA, S. C.; LOPES, C. E. F.; CUNHA, D. M. Integração do quiz como ferramenta de aprendizagem numa disciplina de astronomia na modalidade a distância. In: Simpósio Nacional de Educação em Astronomia, 1., 2011, Rio de Janeiro. Anais. Rio de Janeiro: Sociedade Astronômica Brasileira/UFRJ, 2011, p. 01-07.

GHENSEV, R. O. Uso dos Games na Educação. Dissertação de mestrado - Pós-graduação em Mídias Sociais, Centro Universitário Senac. 55f. São Paulo: SENAC, 2010.

GOMES, T. C. S; MELO, J. C. B. App inventor for android: Uma nova possibilidade para o ensino de lógica de programação. In: Congresso Brasileiro de Informática na Educação, 2., 2013, Campinas. Anais. Campinas: SBC/Unicamp, 2013, p. 620-629.

JEGERS, K. Pervasive game flow: understanding player enjoyment in pervasive gaming. ACM Computers in Entertainment, v. 5, n. 1, p. 1-11, 2007.

KRUGER, F. L; CRUZ, D. M. Os jogos eletrônicos de simulação e a criança. In: XXIV Congresso Brasileiro de Ciência da Comunicação, 24., 2001, Campo Grande. Anais. Campo Grande: Sociedade Brasileira de Estudos Interdisciplinares da Comunicação/UNIDERP, UCDB e UFMS, 2001, p. 01-16.

LEITE, B. S. Gamificando as aulas de química: uma análise prospectiva das propostas de licenciandos em química. RENOTE, v. 15, n. 2, p. 1-10, 2017.

LEITE, B. S. Tecnologias no ensino de química: teoria de prática na formação docente. Curitiba: Appris, 2015.

MARQUES, J. F. Z; MARQUES, K. C. D. A utilização de aplicativos por meio de smartphone como possibilidades para o Ensino de Química. In.: Anais do XVIII ENEQ, Santa Catarina, v. 1, 2016.

MATTAR, J. Games em educação: como os nativos digitais aprendem. São Paulo: Pearson Prentice Hall, 2010.

NICHELE, A. G; SCHLEMMER, E. Aplicativos para o ensino e aprendizagem de Química. RENOTE, v. 12 n. 2, p. 1-9, 2014.

PRENSKY, M. Digital game-based learning. Computers in Entertainment (CIE), v. 1, n. 1, p. 21-21, 2003.

SANTOS, W; ALVES, L. DOM-Um Jogo Sobre Funções Quadráticas: Entre A Educação e o Entretenimento. RENOTE, v. 14, n. 2, p. 1-12, 2016.

SILVA, F. C. V; CAMPOS, A. F; ALMEIDA, M. A. V. Concepções alternativas de licenciandos em Química sobre radioatividade. Experiências em Ensino de Ciências v. 8, n. 1, p. 87-97, 2013.

SOUZA, J. I. R.; LEITE, Q. S. S.; LEITE, B. S. Avaliação das dificuldades dos ingressos no curso de licenciatura em Química no sertão pernambucano. Revista Docência do Ensino Superior, v. 5, p. 135-160, 2015.

TAROUCO, L. M. R; ROLAND, L. C.; FABRE, M. C. J. M.; KONRATH, M. L. P. Jogos educacionais. RENOTE, v. 2, n. 1, p. 1-7, 2004.

TEKIN, B. B; NAKIBOGLU. C. Identifying students' misconceptions about nuclear chemistry. A study of Turkish high school students. Journal of chemical Education, v. 83, n. 11, p. 1712-1718, 2006. 